\title{
Erratum to: Computational Challenges in Modeling and Simulation
}

Christopher Carothers, Alois Ferscha, Richard Fujimoto, David Jefferson, Margaret Loper, Madhav Marathe, Pieter Mosterman, Simon J.E. Taylor and Hamid Vakilzadian

\section{Erratum to:}

Chapter 4 in: R. Fujimoto et al. (eds.), Research Challenges in Modeling and Simulation for Engineering Complex Systems, Simulation Foundations, Methods and Applications, DOI 10.1007/978-3-319-58544-4_4

In the original version of the book, the contributor name and the corresponding affiliation have to be included in Chapter 4. The erratum chapter and the book have been updated with the change. 